\title{
TITLE:
}

\section{Dominating factor of strain-induced crystallization in natural rubber}

$\operatorname{AUTHOR}(S)$ :

Gros, Alice; Tosaka, Masatoshi; Huneau, Bertrand;

Verron, Erwan; Poompradub, Sirilux; Senoo, Kazunobu

CITATION:

Gros, Alice ...[et al]. Dominating factor of strain-induced crystallization in natural rubber. Polymer 2015, 76: 230-236

ISSUE DATE:

2015-10

URL:

http://hdl.handle.net/2433/202579

RIGHT:

(C) 2015. This manuscript version is made available under the CC-BY-NC-ND 4.0 license

http://creativecommons.org/licenses/by-nc-nd/4.0/; The full-text file will be made open to the public on 1 October 2017 in accordance with publisher's 'Terms and Conditions for Self-Archiving'.; この論文は出版社版でありません。引用の際 には出版社版をご確認ご利用ください。; This is not the published version. Please cite only the published version. 


\section{Dominating Factor of Strain-induced Crystallization in Natural Rubber}

Alice Gros ${ }^{\dagger}$, Masatoshi Tosaka ${ }^{\ddagger *}$, Bertrand Huneau ${ }^{\dagger}$, Erwan Verron ${ }^{\dagger}$, Sirilux Poompradub ${ }^{\S}$, and Kazunobu Senoo"

${ }^{\dagger}$ Ecole Centrale de Nantes, Institut de Recherche en Génie Civil et Mécanique (GeM), UMR CNRS 6183, BP 92101, Nantes Cedex 3, France

${ }^{\ddagger}$ Institute for Chemical Research, Kyoto University, Gokasho, Uji, Kyoto-fu 611-0011, Japan.

${ }^{\S}$ Department of Chemical Technology, Faculty of Science, Chulalongkorn University, Phayatai Rd. Patumwan Bangkok 10330, Thailand.

"Corporate R\&D Center, Sumitomo Bakelite Co., Ltd., 1-1-5 Murotani, Nishi-ku, Kobe, Hyogo 6512241, Japan 


\begin{abstract}
:
The contribution of entropy change due to stretching of polymer chains in promoting crystal nucleation is theoretically derived for strain-induced crystallization of natural rubber. The results of theoretical calculation are compared with experimental results obtained by fast time-resolved wide-angle X-ray diffraction. Usual values of surface free energies corresponding to chain-folded nuclei lead to theoretical results far from experimental measurements. Because the discrepancy comes from the large activation energy of nucleation even after the stretching of polymer chains, additional contribution of reduced surface free energies due to the formation of bundle-like nuclei was taken into account. This treatment allows to faithfully reproduce experimental results and then to conclude that nuclei formed in natural rubber during stretching are of bundle-like type. Moreover, it reveals that surface energies have a greater effect on the decrease of critical free energy than the change in entropy due to deformation.
\end{abstract}




\section{Introduction}

Crystallization of oriented polymer chains induced by flow or stretching is an important issue in engineering because resulting changes in morphology strongly affect the properties (mechanical ones for example) of polymer materials. However, the theoretical treatment of crystallization kinetics under molecular orientation is still not successful.

A seminal theory describing the effect of chain stretching was first derived by Flory [1] considering strain-induced crystallization in rubber networks. Since then, other approaches for straininduced crystallization of rubber have been investigated [2-5]. These theories focus on systems at equilibrium, but they hardly deal with crystallization kinetics. The first work devoted to the kinetics of crystallization of oriented polymer melt was proposed by Kobayashi and Nagasawa [6]; it incorporates the rubber elasticity into the nucleation theory developed by Hoffman and coworkers [7,8]. Later, Bushman and McHugh [9] derived a more advanced model considering the formalism of irreversible thermodynamics. In all these works, the emphasis is laid on the decrease in entropy of stretched amorphous chains and on the resulting increase in melting temperature causing the acceleration of crystallization. As a different approach, some other researchers proposed that the change in orientation, rather than the stretch of polymer chains, is the main factor for the acceleration of crystallization [10,11]. To the authors' knowledge, these two points of view have not been considered simultaneously to explain experimental results. Furthermore, formation of characteristic morphologies such as shish-kebab structure $[12,13]$ has not been related to these theories.

In the present paper, we evaluate the contribution of entropy change due to stretching of polymer chains in promoting crystal nucleation in cross-linked natural rubber (NR) and demonstrate that usual thermodynamic parameters cannot explain the experimentally observed dependence of crystallization rate on stretch ratio. Then we introduce additional contribution of reduced surface free energies due to the formation of bundle-like nuclei to explain the observed tendencies. The implication of smaller surface free energies for this type of nuclei than for chain-folded nuclei is finally discussed. 


\section{Experimental}

Recently, studies on kinetics of strain-induced crystallization of cross-linked NR by wide-angle X-ray diffraction (WAXD) have been reported [14-17]. The details of the experiments, similar to those of ref. [14], are given below.

\subsection{Materials}

Sheets (1 mm or $2 \mathrm{~mm}$ thick) of vulcanized NR were prepared. The recipes for the preparation of the samples and the cure conditions are listed in Table 1. Ring-shaped specimens were die-cut from the sample sheets. The width and circumference of the specimens were ca. $1 \mathrm{~mm}$ and $50 \mathrm{~mm}$, respectively. The initial length corresponds to the half of the circumference (i.e. $25 \mathrm{~mm}$ ).

Table 1. Recipes and densities of cross-linked NR samples

\begin{tabular}{|c|c|c|c|c|c|c|c|}
\hline $\begin{array}{c}\text { Sample } \\
\text { code }\end{array}$ & $\begin{array}{l}\mathrm{NR}^{\mathrm{a}} \\
\text { (part) }\end{array}$ & $\begin{array}{c}\text { Stearic acid } \\
\text { (part) }\end{array}$ & $\begin{array}{l}\text { ZnO } \\
\text { (part) }\end{array}$ & $\begin{array}{l}\text { CBS }^{b} \\
\text { (part) }\end{array}$ & $\begin{array}{l}\text { Sulfur } \\
\text { (part) }\end{array}$ & $\begin{array}{l}\text { Curing time } \\
\text { (min) }\end{array}$ & $\begin{array}{l}\text { Network-chain } \\
\text { density }{ }^{d}\left(m^{-3}\right)\end{array}$ \\
\hline NR-S1.125 & 100 & 2 & 1 & 0.75 & 1.125 & 35 & $5.03 \times 10^{25}$ \\
\hline NR-S2.25 & 100 & 2 & 1 & 1.5 & 2.25 & 25 & $8.41 \times 10^{25}$ \\
\hline NR-S4.5 & 100 & 2 & 1 & 3 & 4.5 & 20 & $12.5 \times 10^{25}$ \\
\hline
\end{tabular}

${ }^{\mathrm{a}}$ RSS No.1

${ }^{\mathrm{b}} \mathrm{N}$-cyclohexyl-2-benzothiazole sulfenamide, curing temperature with sulfur $140^{\circ} \mathrm{C}$

${ }^{\mathrm{c}}$ Network-chain density estimated from the initial slope of the stress-strain curve on the basis of the rubber elasticity theory [18]

\subsection{WAXD experiments}

The WAXD experiments were performed at BL-40XU beam line in SPring-8, Japan. The wave length was $0.0832 \mathrm{~nm}(15 \mathrm{keV})$ and the camera length was ca. 125 - $140 \mathrm{~mm}$. The specimen temperature was $302 \mathrm{~K}$. The drawing axis of the specimen was tilted to adjust the 002 reflection to satisfy the Bragg reflection condition. The two-dimensional WAXD patterns were recorded every 36 
ms using a Hamamatsu C4880-80 CCD camera. A custom-made tensile tester [14], which enabled WAXD analysis of a fixed part of the specimen was placed on the beam line. The specimen was

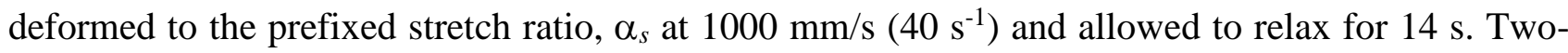
dimensional (2D) WAXD patterns were recorded during and after deformation. The origin (0 s) of the elapsed time $t$ is defined at the cessation of the deformation.

\subsection{Processing of the WAXD data}

Equatorial intensity distribution was obtained from the 2D WAXD data using Fit2D software (European Synchrotron Radiation Facility). Then the equatorial intensity distribution was decomposed into linear background, 200 and 120 crystalline reflections and amorphous halo by fitting with Voigt functions using Fityk [19] (peak fitting software) in combination with home-made software to generate automatic execution script. Figure 1 shows an example of the decomposition of the intensity distribution. Relative intensity of the 200 reflection, $I(t)$, as a function of elapsed time, $t$, was calculated from the results of fitting. That is to say,

$$
I(t)=\frac{I_{200}(t)}{I_{200}(t)+I_{120}(t)+I_{\text {amorphous }}(t)}
$$

where $I_{\mathrm{x}}(t)$ indicates the integrated intensity of the reflection or halo designated by the subscript $\mathrm{x}$.

Then $I(t)$ was fitted using the formula [14];

$$
I(t)=I_{0}+I_{f}\left[1-\exp \left(\frac{t}{\tau_{f}}\right)\right]+I_{s}\left[1-\exp \left(\frac{t}{\tau_{s}}\right)\right]
$$

where $\tau_{f}$ and $\tau_{s}$ are the time constants of the crystallization processes $\left(\tau_{f}<\tau_{s}\right)$; $I_{f}$ and $I_{s}$ are, respectively, the amplitude of these processes and $I_{0}$ is a constant related to the initial value. An example of the time-dependent change of $I(t)$ and its regression curve are shown in Figure 2. As has been described in ref. [14], time constants do not show definite dependence on the stretch ratio $\alpha_{s}$. Considering the experimental error, time constants were regarded as unchanged values, independent of $\alpha_{s}$. In this case, 
each of $I_{0}, I_{f}$ and $I_{s}$ is thought to be proportional to crystallization rate. In a previous study, Tosaka et al. obtained linear dependence of $I_{0}, I_{f}$ and $I_{s}$ on $\alpha_{s}$ [14]. Therefore, their summation, $I_{\text {sum, was }}$ evaluated as a measure of crystallization rate.

In the equatorial intensity distribution, 200 reflection was fitted again with Gaussian function along with linear background. Then crystallite size was estimated by using the Scherrer equation:

$$
L_{h k l}=K \cdot \lambda /(\beta \cdot \cos \theta)
$$

where $L_{h k l}$ is the crystallite size in the direction perpendicular to the $(h k l)$ plane, $\lambda$ is the wavelength, $\beta$ the half width estimated by the Gaussian fitting and $\theta$ is the Bragg angle (half of the scattering angle). The value 0.89 was used for $K[20]$.

\subsection{Tensile measurement}

A conventional tensile tester (Shimadzu Autograph AGS-1kNG) was used for the uniaxial tensile measurements. The specimens were stretched at $25 \mathrm{~mm} / \mathrm{min}$ at $302 \mathrm{~K}$. The tensile force was recorded every $0.5 \mathrm{~s}$.

\subsection{Experimental results}

Figure 3 shows results of time-resolved WAXD analysis of strain-induced crystallization. We consider here four main features related to the kinetics of strain-induced crystallization: first the linear relationship between crystallization rate and stretch ratio in the studied interval of stretch ratio (Figure 3a), second the small dependence of the crystallization rate on network-chain density (that is to say, among the samples), third the small increase in crystallite size during its time evolution (Figure 3b), and fourth the decrease in crystallite size with the increase in crosslinking density (Figure 3c). Invoking the latter two features, it can be stated that once a crystal nucleus is formed, it grows quickly to its maximal size, which is restricted by available space between crosslinks. Therefore, the rate of strain-induced crystallization can be approximated to be proportional to the nucleation rate, and then invoking the first feature, the nucleation rate is linearly related to the stretch ratio for the studied stretch 
ratios. Considering the crystal morphology [21-23] of strain-induced crystals of NR, this approximation should not induce severe errors as large as several orders of magnitude.

\section{Theoretical Estimation}

\subsection{Critical Gibbs free energy of nucleation}

For the cross-linked nature of rubber, we consider that the local stretch at chain scale is equal to the one applied to the macroscopic sample (affine assumption). Thus, nucleation rates issued from the rubber elasticity theory [18] can be compared with experimental (macroscopic) results. Our theoretical treatment for the evaluation of the effect of entropy change follows Flory's idea [1] and is similar to those of precedent studies [24-26]. Let us consider the change in Gibbs free energy $\Delta G$ due to the formation of a parallelepipedic crystal of dimensions $L_{1}$ (height in the $c$ direction), $L_{2}$ and $L_{3}\left(L_{2}=L_{3}\right)$ [27]:

$$
\Delta G=2 L_{2}^{2} \sigma_{e}+4 L_{1} L_{2} \sigma+L_{1} L_{2}^{2} \Delta F
$$

where $\sigma_{e}$ is the end surface free energy (at the top and bottom surfaces of the crystallite) per unit area, $\sigma$ is the side surface free energy and $\Delta F$ stands for the change in bulk free energy per unit volume assuming an infinitely large crystal. By solving the conditions for

$$
\frac{d \Delta G}{d L_{1}}=0, \frac{d \Delta G}{d L_{2}}=0
$$

which give the critical values for the nucleus to be able to grow, we obtain the critical sizes of primary nucleus:

$$
L_{1}^{*}=-\frac{4 \sigma_{e}}{\Delta F}, \quad L_{2}^{*}=-\frac{4 \sigma}{\Delta F}
$$

and the resulting Gibbs free energy for the formation of critical nucleus: 


$$
\Delta G^{*}=2 L_{2}^{* 2} \sigma_{e}+4 L_{1}^{*} L_{2}^{*} \sigma+L_{1}^{*} L_{2}^{* 2} \Delta F=32 \frac{\sigma^{2} \sigma_{e}}{\Delta F^{2}}
$$

where $\sigma_{e}$ is the end surface free energy (at the top and bottom surfaces of the crystallite) per unit area, $\sigma$ is the side surface free energy and $\Delta F$ stands for the change in bulk free energy per unit volume assuming an infinitely large crystal. Following Flory’s idea [1], $\Delta F$ is the key factor that is responsible for strain-induced crystallization. In the case of stretched amorphous material, $\Delta F$ is given by:

$$
\Delta F=\Delta H-T \Delta S=\Delta H-T\left(\Delta S_{0}-\Delta S_{d e f}\right)
$$

with $T$ the temperature, $\Delta S$ the total entropy change of crystallization, $\Delta S_{0}$ the entropy change of crystallization of the unstretched amorphous material and $\Delta S_{\text {def }}$ the entropy change due to straining of amorphous chains (equal to 0 when chains are unstrained). $\Delta H$ is the melting enthalpy, supposed to be independent of strain $[5,24]$. From the expression of equilibrium melting temperature of unstrained polymer, $T_{m}^{0}$, we get:

$$
\Delta S_{0}=\frac{\Delta H}{T_{m}^{0}}
$$

therefore

$$
\Delta F=\frac{T_{m}^{0}-T}{T_{m}^{0}} \Delta H+T \Delta S_{d e f}
$$

For readers' convenience, setting $\Delta F=0$ in Eq. (8) and comparing with Eq. (9) leads to

$$
T_{m, \alpha}^{0}=\frac{\Delta H}{\Delta S_{0}-\Delta S_{\text {def }}}>\frac{\Delta H}{\Delta S_{0}}=T_{m}^{0}
$$

which indicates the elevation in equilibrium melting temperature, $T_{m, \alpha}^{0}$, of stretched polymer. 
$\Delta S_{d e f}$ is equal to the difference between the entropy of an unstrained material, $S(1)$, and the entropy at a given stretch ratio $\alpha, S(\alpha)$. This entropy can be calculated as follows [18]. From the first law of thermodynamics, the change in internal energy $\mathrm{d} U$ in a reversible process is given as:

$$
\mathrm{d} U=\mathrm{d} Q+\mathrm{d} W=T \mathrm{~d} S+\mathrm{d} W
$$

where $d Q$ and $d W$ are respectively heat absorbed by the system and the work of external forces. The change in Helmholtz free energy $\mathrm{d} A$ for a system in equilibrium under elastic deformation is:

$$
\mathrm{d} A=\mathrm{d} U-T \mathrm{~d} S
$$

Combining Eqs. (12) and (13), we obtain for constant-volume condition:

$$
\mathrm{d} A=\mathrm{d} W=f \mathrm{~d} \alpha
$$

where $f$ is tensile stress and $\alpha$ is the stretch ratio. From Eqs. (13) and (14), the tensile stress is expressed as follows:

$$
f=\left(\frac{\partial W}{\partial \alpha}\right)_{T}=\left(\frac{\partial A}{\partial \alpha}\right)_{T}=\left(\frac{\partial U}{\partial \alpha}\right)_{T}-T\left(\frac{\partial S}{\partial \alpha}\right)_{T}
$$

Indeed, we can reasonably assume that there is no volume change of the rubber sample upon stretching. Additionally, we can assume that the deformation of rubber at constant temperature is associated with a reduction of entropy, with no change in internal energy. Under this assumption, the entropy can be calculated from the integration of the nominal stress $f$ of network [18]:

$$
S(\alpha)=-\frac{1}{T} \int f d \alpha
$$

therefore

$$
\Delta S_{\text {def }}=S(\alpha)-S(1)=-\frac{1}{T} \int_{1}^{\alpha} f(x) d x
$$

By combining Eqs. (7), (8) and (17), the Gibbs free energy for the formation of a critical nucleus, $\Delta G^{*}$, can be expressed as a function of stretch ratio $\alpha$. For its numerical calculation, 
$\int_{1}^{\alpha} f(x) d x$ is evaluated by the integration of the experimental stress-strain curve of each sample (Figure 4).

\subsection{Rate of primary nucleation}

Besides, the rate of primary nucleation, $I$, at constant temperature is written as [28]:

$$
I_{\text {sum }}=I_{0} \exp \left(-\frac{\Delta G^{*}}{k T}\right)
$$

where $I_{0}$ is a constant. Eqs. (7) and (18) finally lead to

$$
I_{\text {sum }}=I_{0} \exp \left(-\frac{32 \sigma^{2} \sigma_{e}}{k T \Delta F^{2}}\right)
$$

The contribution of entropy change due to stretching of polymer chains can be directly derived in this way. The values of the necessary thermodynamic parameters are listed in Table 2.

Table 2. Values used for calculations and plots for chain-folded nucleus of NR

\begin{tabular}{lccc}
\hline & Symbol & Value & Ref. \\
Boltzmann constant & $k$ & $1.38 \times 10^{-23} \mathrm{~J} \mathrm{~K}^{-1}$ & - \\
Temperature & $T$ & $302 \mathrm{~K}$ & - \\
Equilibrium melting temperature & $T_{m}^{0}$ & $309 \mathrm{~K}$ & {$[29]$} \\
Melting enthalpy & $\Delta H$ & $-5.99 \times 10^{7} \mathrm{~J} \mathrm{~m}^{-3}$ & {$[30]$} \\
Side surface free energy & $\sigma$ & $0.013 \mathrm{~J} \mathrm{~m}^{-2}$ & {$[29]$} \\
End surface free energy & & $0.024 \mathrm{~J} \mathrm{~m}^{-2}$ & [29] \\
& $\sigma_{\mathrm{e}}$ & & \\
\hline
\end{tabular}

\section{Discussion}

\subsection{Comparison with experimental data}


When usual values of $\sigma$ and $\sigma_{\mathrm{e}}$ corresponding to chain-folded nuclei are considered (Table 2), the dependence of $I_{\text {sum }} / I_{0}=\exp \left(-\Delta G^{*} / k T\right)$ on $\alpha$ shows a drastic increase of the nucleation rate, as shown in Figure 5. The ordinate in linear scale (Figure 5a) allows to distinguish the result for only one sample with a very steep slope around $\alpha=8$ because the increase is of several orders of magnitude. The results for other samples lie near 0 on the graph. With a semilogarithmic scale representation (Figure 5b), we notice a strong dependence of nucleation rate on stretch ratio for all the samples, and at the same time, on network-chain density, which differs among the samples (see last row of Table 1). These features are considerably different from experimentally measured crystallization rate of NR (Figure 3a), and this inconsistency is too large to be solely attributed to the assumption of proportionality between growth rate and primary nucleation rate.

Figure 6 shows the calculated dependence on $\alpha$ of activation energy of nucleation $\Delta G^{*}$ using the values given in Table 2, and $k T\left(4.17 \times 10^{-21} \mathrm{~J}\right.$ at $\left.302 \mathrm{~K}\right)$. As expected, $\Delta G^{*}$ decreases with increasing $\alpha$. However, even at $\alpha=8$, around which NR samples sometimes come to rupture, the absolute value of $\Delta G^{*}$ is larger than $10^{-19} \mathrm{~J}$, which is three or more orders larger than $k T$. Thus the large dependence of nucleation rate on $\alpha$ (Figure 5) comes from the large variations (from around 25 to $2 \times 10^{4}$ ) of $\Delta G^{*} / k T$ in the exponential function. In the first place, Eq. (18), which is of Arrhenius type, tells us that nucleation and subsequent crystallization will hardly occur when $\Delta G^{*}$ is too large compared to $k T$. The experimental fact that crystallization occurs and the mild dependence of crystallization rate on stretch ratio (Figure 3a) suggest that $\Delta G^{*}$ is overestimated. Consequently, we have to consider other effects reducing $\Delta G^{*}$ to account for experimental facts. According to Eq. (7), $\Delta G^{*}$ is determined by the bulk free energy $\Delta F$, and surface free energies, $\sigma$ and $\sigma_{\mathrm{e}}$. In the calculation of $\Delta F$, the effect of chain stretching is already introduced and no additional change can be considered. The remaining parameters that can reduce $\Delta G^{*}$ are inevitably the surface free energies. Different values of surface free energies imply that nuclei have different surface structure from the above 
considered folded-chain nuclei. In case of strain-induced crystallization, such structure is reasonably attributed to the bundle-like one without chain folding, and to parallel orientation of the chains in the nuclei and surrounding amorphous chains. This morphology is also the one considered in Flory's basic models [1]. Even when the morphological model of nuclei is changed, the theoretical treatment [31] is essentially the same as described above.

\subsection{Estimation of surface energies for SIC in NR}

The bundle-like nucleus considered in strain-induced crystallization of NR is expected to have smaller $\sigma_{\mathrm{e}}$ because the work for chain folding is not consumed for the formation of the end surface.

In the case of polyethylene (PE), theoretically estimated $\sigma_{e}$ for a bundle-like nucleus is 0.009 $\mathrm{J}^{-\mathrm{m}^{-2}}$ [27], which is $1 / 10$ of the corresponding chain-folded nucleus $\left(0.09 \mathrm{~J}^{-\mathrm{m}^{-2}}\right)$ [8]. Indeed, Yamazaki et al. [32] report smaller $\sigma_{\mathrm{e}}$ for bundle-like nuclei created in oriented melt of isotactic polypropylene (iPP) and PE than for chain-folded nuclei. Furthermore, Lu et al. [33] show that iPP crystal with less chain folding has smaller fold surface free energy on crystallization.

Moreover, Coppola et al. [11] calculate the reduction of free energy by flow-induced chain orientation. In the current study, nuclei are also surrounded by oriented amorphous, and a reduction of free energy can be attributed to a smaller $\sigma$ (Eqs. (4) and (5)). This reduction of $\sigma$ is also reported by Yamazaki et al. for iPP and PE [32]. To this extent, effect of the orientation can be reasonably incorporated into the numerical calculation using Eq. (19) as the reduction of surface free energies, $\sigma$ and $\sigma_{\mathrm{e}}$, by the formation of bundle-like nuclei.

Currently, values of surface free energies for bundle-like nuclei of NR are not established. We therefore estimated the product of surface free energies, $\sigma^{2} \sigma_{e}$, from experimental crystallization rate (which is assumed to be proportional to the nucleation rate) by fitting with Eq. (19) under the assumption that $\sigma^{2} \sigma_{e}$ is constant for each sample. The results of fitting are shown in Figure 7 and the estimated values of $\sigma^{2} \sigma_{e}$ are reported in Table 3. Compared to $4.056 \times 10^{-6} \mathrm{~J}^{3} \mathrm{~m}^{-6}$ for chain-folded nuclei (issued from Table 2), experimentally obtained values of $\sigma^{2} \sigma_{e}$ from the stretched samples are 
approximately 400 to 1520 times smaller (Table 3) and show a dependence to network-chain density. These ratios of reduction are of the same order of magnitude as those between bundle-like and chainfolded nuclei for iPP and PE reported by Yamazaki et al. [32]. On the basis of this consistency, we conclude that nuclei formed in strain-induced crystallization are of bundle-like type. The fluctuation of $\sigma^{2} \sigma_{e}$ in Table 3 is suspected to come mainly from the degree of orientation of amorphous chains in which nuclei are embedded.

Table 3. Values of fitted $\sigma^{2} \sigma_{\mathrm{e}}$ for NR samples

\begin{tabular}{cccc}
\hline Sample & NR-S1.125 & NR-S2.25 & NR-S4.5 \\
\hline$\sigma^{2} \sigma_{\mathrm{e}}$ for bundle-like nucleus $\left(\mathbf{J}^{\mathbf{3}} \mathbf{~ m}^{-\mathbf{6}}\right)$ & $2.67 \times 10^{-9}$ & $3.62 \times 10^{-9}$ & $9.84 \times 10^{-9}$ \\
$\left(\sigma^{2} \sigma_{\mathrm{e}}\right)_{\text {bundle }} /\left(\sigma^{2} \sigma_{\mathrm{e}}\right)_{\text {folded }}$ & $1 / 1519$ & $1 / 1120$ & $1 / 412$ \\
\hline
\end{tabular}

Figure 8 shows the same plots as Figure 6, on which the new $\Delta G^{*}$ calculated with the fitted values of $\sigma^{2} \sigma_{\mathrm{e}}$ (Table 3) have been superimposed. The reduction of $\sigma^{2} \sigma_{\mathrm{e}}$ implies that $\Delta G^{*}$ is also reduced by two or three orders of magnitude, while entropy change due to chain stretching divides $\Delta G^{*}$ only by $5 \sim 20$ from the unstretched state to $\alpha=4$ (at which crystallization begins [34-37]). These numerical estimations allow us to argue that reduction of surface free energy by the formation of bundle-like nuclei is the dominating factor in strain-induced crystallization of natural rubber. This argument is partly in agreement with previous works $[10,11]$ in which orientation of polymer chains is considered to be the main factor for the acceleration of crystallization. However, the effects on the surface free energies have not been considered before.

Besides, smaller surface free energies of bundle-like nuclei should not be limited to cross-linked NR, considering the study by Yamazaki et al. [32]. Thus, it is strongly presumed that the reason why the previous theoretical treatments failed to explain some experimental results is this missing of the 
effect of changes in surface free energies. Hereafter, we further discuss the implication of smaller surface free energy of bundle-like nuclei for the formation of the shish-kebab structure in linear polymer.

\subsection{Formation mechanism of shish-kebab structure in linear polymer}

The formation process of the shish part of shish-kebab structure have been explained by chain extension caused by flow field [13]. However, growth of shish of isotactic polystyrene (iPS) crystal in the absence of flow field, which is inconsistent with the original model of the shish formation, has been reported by Petermann and coworkers $[38,39]$. Here, we can propose alternative model of shish formation which can explain the Petermann's observation, considering the large difference in $\sigma_{\mathrm{e}}$ between chain-folded and bundle-like nucleus. Once oriented zone is generated in polymer melt by application of stretching or shear, bundle-like nuclei are preferentially formed as they are more stable than chain-folded nuclei. These bundle-like nuclei tend to keep the unfolded end surfaces because the transformation into folded surfaces will considerably increase $\sigma_{\mathrm{e}}$. As long as local orientation of amorphous chains ahead of the growth front (end surface) is parallel to the growing direction of the bundle-like crystals, such growth continues and consequently, fibrillar shish structures are formed. Here we assume that bundle-like crystals are of very thin, limited sizes, otherwise the amorphous chains near the bundle-like boundaries would be overcrowded and the bundle-like interface would become unstable.

On the basis of this idea, Petermann's observation for shish-kebab growth of iPS is explained as follows: in the case of iPS, work of chain folding $\left(7.1 \mathrm{kcal}^{\mathrm{mol}}{ }^{-1}\right)$ is larger than the one of PE (4.9 kcal.mol ${ }^{-1}$ ) [40]. Therefore, when the shish is going to grow under sufficient supercooling, the bundlelike form may be conserved, even when the growth front is surrounded by isotropic amorphous.

\section{Conclusion}


The free energy of nuclei in strain-induced crystallization of natural rubber has been estimated. Results assuming chain-folded nuclei are far different from experimental ones, and accordingly, the reduction of free energy due to the orientation of the stretched chains has to be taken in account. The reduction of free energy has been reasonably attributed to the formation of bundle-like nuclei. From the comparisons of numerical estimations with experimental data, smaller surface free energies of bundle-like nuclei are revealed to have a dominant effect on the reduction of the activation energy of nucleation. This idea of modification of surface energy is believed to contribute to overcome the failure of previous theoretical treatments [41] and bring a great progress in the understanding and theoretical derivation of crystallization in natural rubber, and more generally in oriented polymers. Particularly, this concept would also explain the preferential formation of shish part in the shish-kebab structure in isotropic amorphous.

\section{ACKNOWLEDGMENT}

This work was partly supported by ICR-KU International Short-term Exchange Program for Young Researchers. The synchrotron WAXD experiments at the SPring-8 were performed under the approval of the Japan Synchrotron Radiation Research Institute (JASRI) (Proposal No. 2013A1203, 2014A1118).

\section{REFERENCES}

[1] Flory P J. J Chem Phys 1947; 15: 397-408.

[2] Gaylord R J. J Polym Sci Polym Phys Ed 1976; 14: 1827-1837.

[3] Gaylord R J, Lohse D J. Polym Eng Sci 1976; 16: 163-167.

[4] Smith Jr. K J. Polym Eng Sci 1976; 16: 168-175.

[5] Yamamoto M, White J L. J Polym Sci Part A-2 1971; 9: 1399-1415.

[6] Kobayashi K, Nagasawa T. J Macromol Sci-Phys 1970; B4: 331-345. 
[7] Hoffman J D, Davis G T, Lauritzen J I J. In: Hannay N B, editors. Treatise on Solid State Chemistry, Vol. 3. New York: Plenum Press, 1976. pp. 497-614.

[8] Hoffman J D, Miller R L. Polymer 1997; 38: 3151-3212.

[9] Bushman A C, McHugh A J. J Polym Sci Part B Polym Phys 1996; 34: 2393-2407.

[10] Andersen P G, Carr S H. Polymer Eng Sci 1978; 18: 215-221.

[11] Coppola S, Grizzuti N, Maffettone P L. Macromolecules 2001; 34: 5030-5036.

[12] Pennings A J, Kiel A M. Kollid Z Z 1965; 205: 160-162.

[13] Pennings A J, van der Mark J M A A, Kiel A M. Kolloid Z Z Polym 1970; 237: 336-358.

[14] Tosaka M, Senoo K, Sato K, Noda M, Ohta N. Polymer 2012; 53: 864-872.

[15] Bruning K, Schneider K, Roth S V, Heinrich G. Macromolecules 2012; 45: 7914-7919.

[16] Albouy P-A, Guillier G, Petermann D, Vieyres A, Sanseau O, Sotta P. Polymer 2012; 53: 3133324.

[17] Candau N, Chazeau L, Chenal J-M, Gauthier C, Ferreira J, Munch E, Rochas C. Polymer 2012; 53: $2540-2543$.

[18] Treloar L R G, The Physics of Rubber Elasticity, Third Edition, Oxford: Clarendon Press, 1975.

[19] Wojdyr M. J Appl Cryst 2010; 43: 1126-1128.

[20] Klug H P, Alexander L E, X-ray Diffraction Procedures for Polycrystalline and Amorphous Materials, 2nd Ed., New York: Wiley-Interscience, 1974.

[21] Walters MH. J Polym Sci Part A 1963; 1: 3091-3103.

[22] Yau W, Stein R S. J Polym Sci B 1964; 2: 231-236.

[23] Yau W, Stein R S. J Polym Sci A-2 1968; 6: 1-30.

[24] Shepherd J E, McDowell D L, Jacob K I. J Mech Phys Solids 2006; 54: 467-489.

[25] Candau N, Laghmach R, Chazeau L, Chenal J-M, Gauthier C, Biben T, Munch E. Macromolecules 2014; 47: 5815-5824. 
[26] Liu D, Tian N, Huang N, Cui K, Wang Z, Hu T, Yang H, Li X, Li L. Macromolecules 2014; 47: 6813-6823.

[27] Hoffman J D, Lauritzen J J I. J Res Natl Bur Stand A Phys Chem 1961; 65A: 297-336.

[28] Turnbull D, Fisher J C. J Chem Phys 1949; 17: 71-73.

[29] Kawahara S, Takano K, Yunyongwattanakorn J, Isono Y, Hikosaka M, Sakdapipanich J T, Tanaka Y. Polymer J 2004; 36: 361-367.

[30] Roberts D E, Mandelkern L. J Am Chem 1955; Soc.77: 781-786.

[31] Mandelkern L, Quinn FA and Flory PJ, J Appl Phys 1954; 25: 830-839.

[32] Yamazaki S, Watanabe K, Okada K, Yamada K, Tagashira K, Toda A, Hikosaka M. Polymer 2005; 46: 1675-1684.

[33] Lu Y, Wang Y, Jiang Z, Men Y. ACS Macro Lett 2014; 3: 1101-1105.

[34] Tosaka M, Kohjiya S, Murakami S, Poompradub S, Ikeda Y, Toki S, Sics I, Hsiao B S. Rubber Chem Technol 2004; 77: 711-723.

[35] Tosaka M, Murakami S, Poompradub S, Kohjiya S, Ikeda Y, Toki S, Sics I, Hsiao B S. Macromolecules 2004; 37: 3299-3309.

[36] Trabelsi S, Albouy P-A, Rault J. Macromolecules 2003; 36: 7624-7639.

[37] Chenal J-M, Chazeau L, Guy L, Bomal Y, Gauthier C. Polymer 2007; 48: 1042-1046.

[38] Petermann J, Miles M, Gleiter H. J Polym Sci Polym Phys Ed 1979; 17: 55-62.

[39] Lieberwirth I, Loos J, Petermann J, Keller A. J Polym Sci Part B Polym Phys 2000; 38: 11831187.

[40] Lauritzen Jr JI, Hoffman JD. J Appl Phys 1973; 44: 4340-4352.

[41] Tosaka M. Macromolecules 2009; 42: 6166-6174. 


\section{Figure Captions}

Figure 1. An example of decomposition of WAXD intensity distribution. A part $\left(q<9 \mathrm{~nm}^{-1}\right)$ of the original data (dotted line) was excluded from the fitting Sample : NR-S1.125, stretch ratio : 6, temperature : $29^{\circ} \mathrm{C}, t: 14 \mathrm{~s}$.

Figure 2. Time-dependent change of experimental $I(t)$ (solid line) and its regression curve (broken line). Sample : NR-S1.125, stretch ratio : 6, temperature : $29^{\circ} \mathrm{C}$.

Figure 3. Results of time-resolved WAXD measurements. (a) Dependence of the total increment of crystallinity index, $I_{\text {sum }}$, on stretch ratio. (b) Time evolution of crystallite size, $L_{200}$. (c) Dependence of crystallite size on network-chain density.

Figure 4. Stress-strain curves of NR samples at $302 \mathrm{~K}$.

Figure 5. Calculated dependence of $I_{\text {sum }} / I_{0}=\exp \left(-\Delta G^{*} / k T\right)$ on stretch ratio $\alpha$ at $302 \mathrm{~K}$ for chain-folded nuclei in linear scale (a) and semilogarithmic scale (b).

Figure 6. Semilogarithmic scale plot of calculated $\Delta G^{*}$ for a chain-folded nucleus as a function of stretch ratio at $302 \mathrm{~K}$.

Figure 7. Dependence of nucleation rate on stretch ratio at $302 \mathrm{~K}$ for bundle-like nuclei: experimental data (unfilled symbols) and fitted theoretical data (lines) with adjusted $I_{0}$ and $\sigma^{2} \sigma_{e}$.

Figure 8. Comparison of $\Delta G^{*}$ with respect to the stretch ratio calculated with values of $\sigma^{2} \sigma_{e}$ for foldedchain and bundle-like nuclei. 
Figure 1

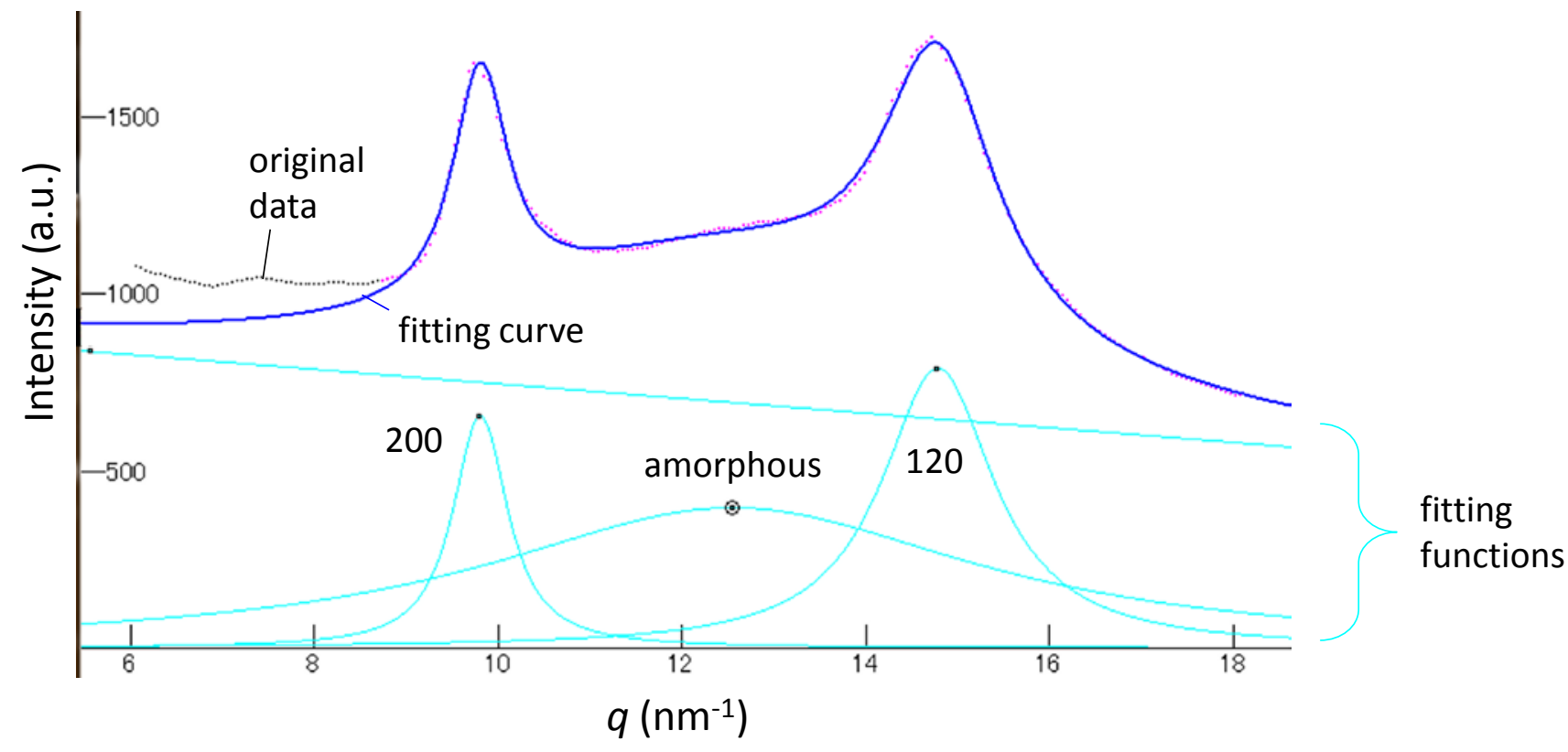




\section{Figure 2}

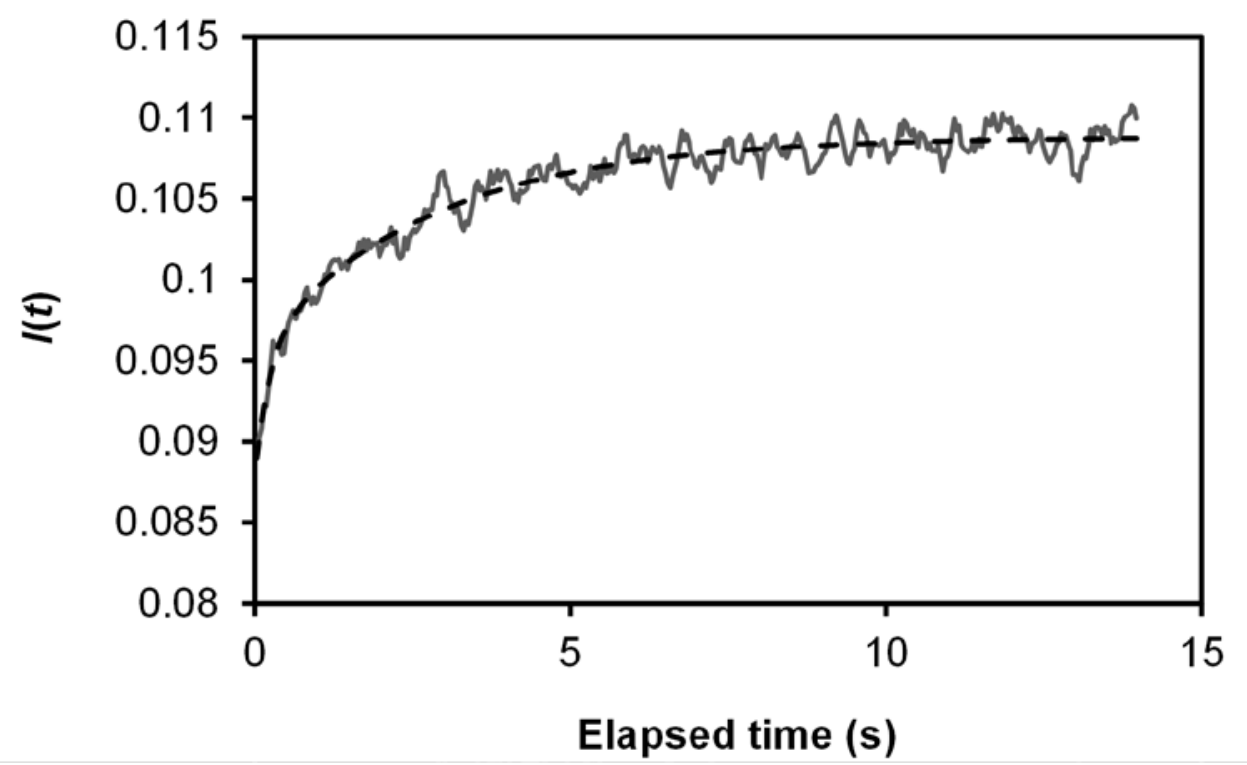



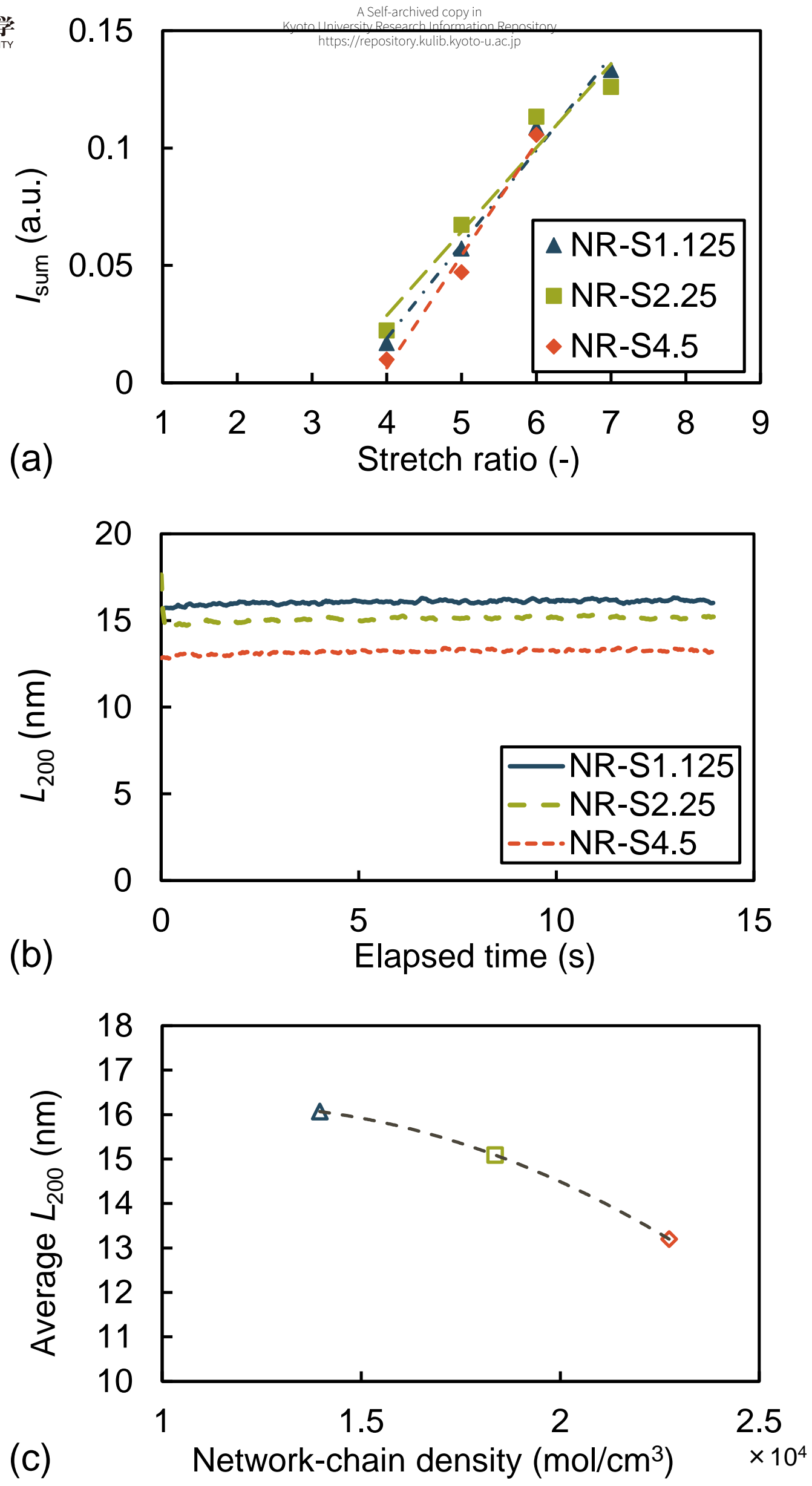


\section{Figure 4}

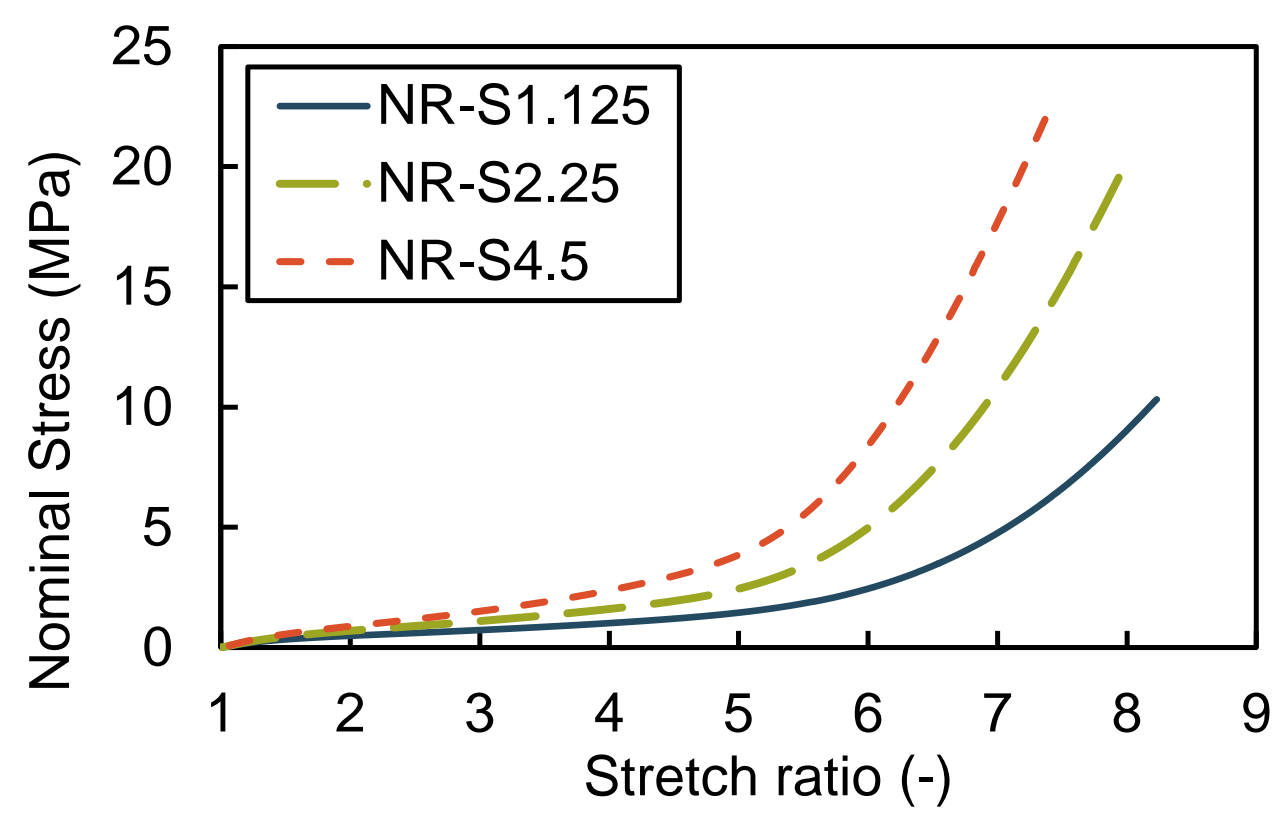




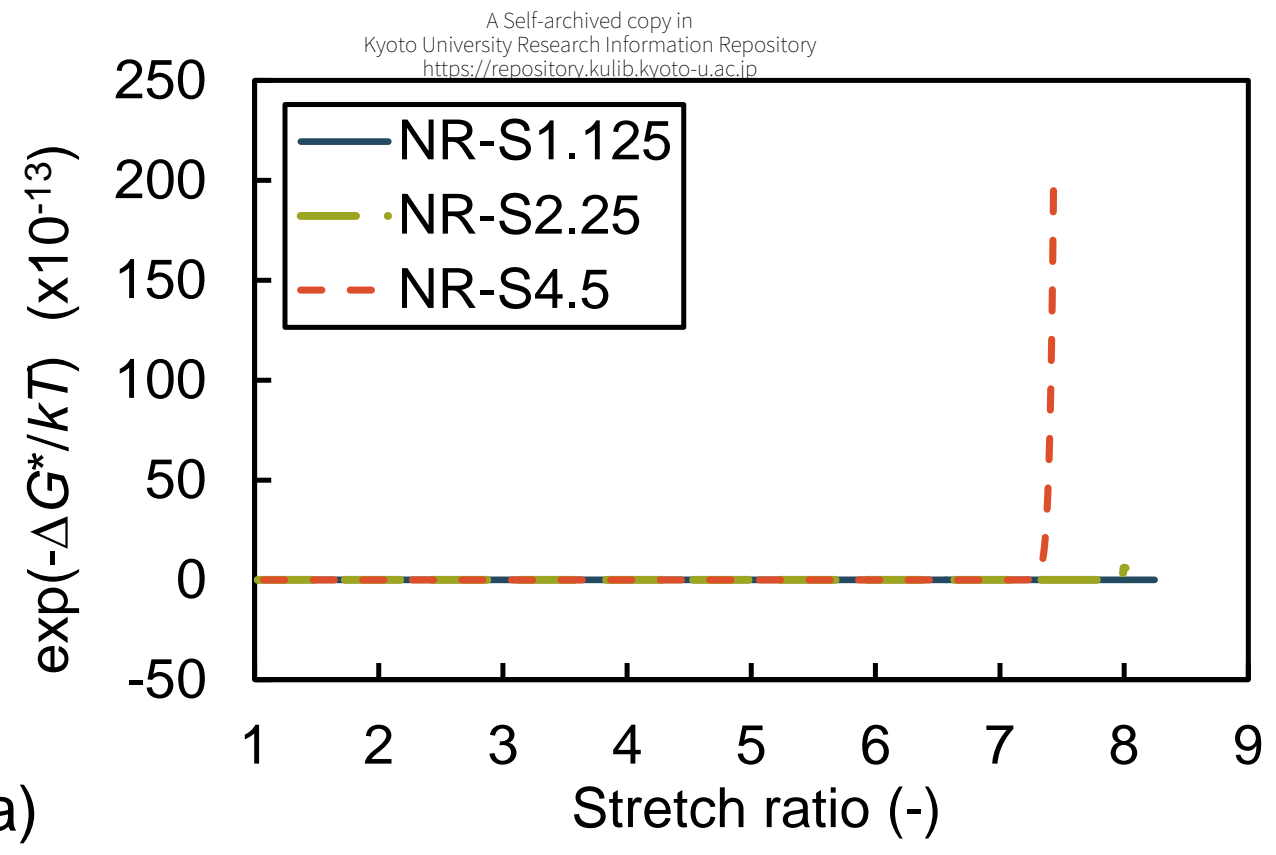

(a)

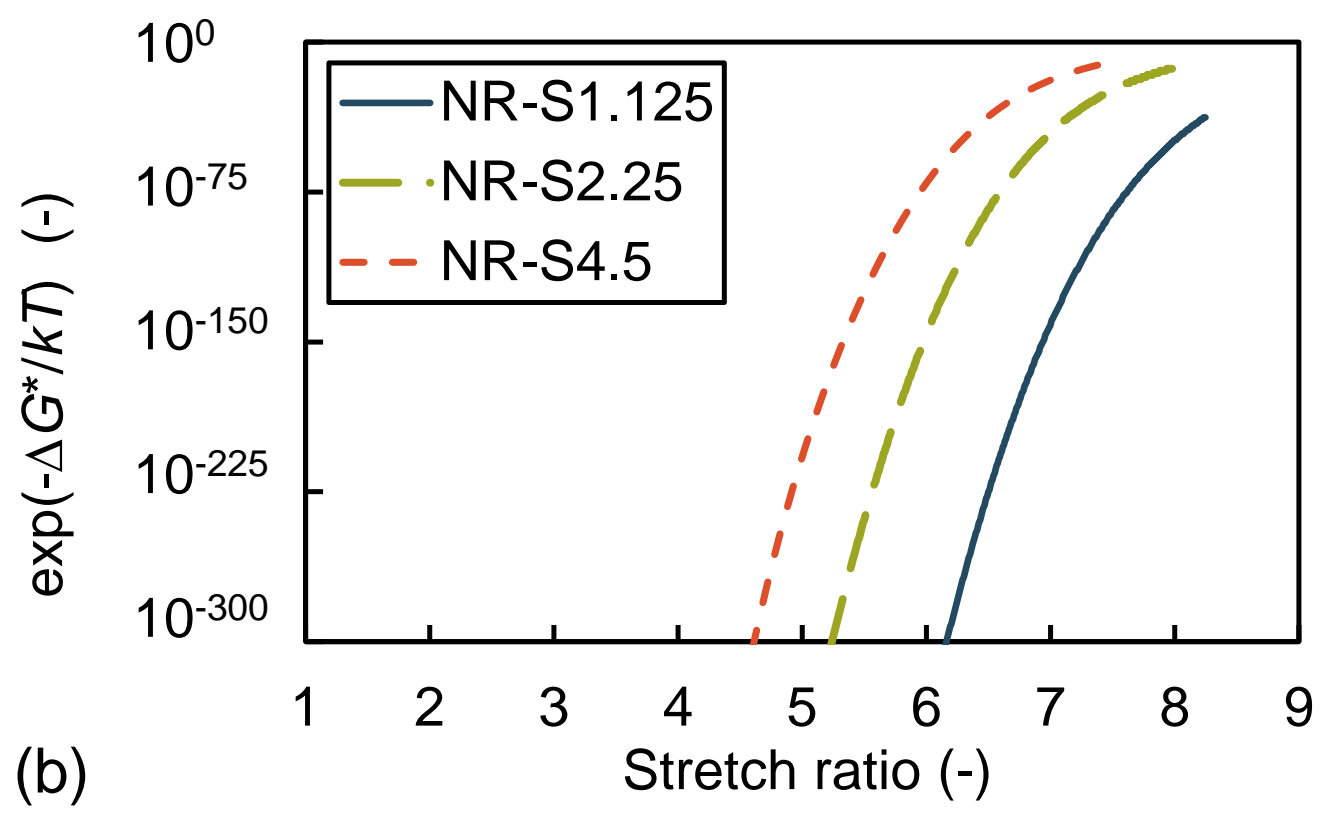


Figure 6

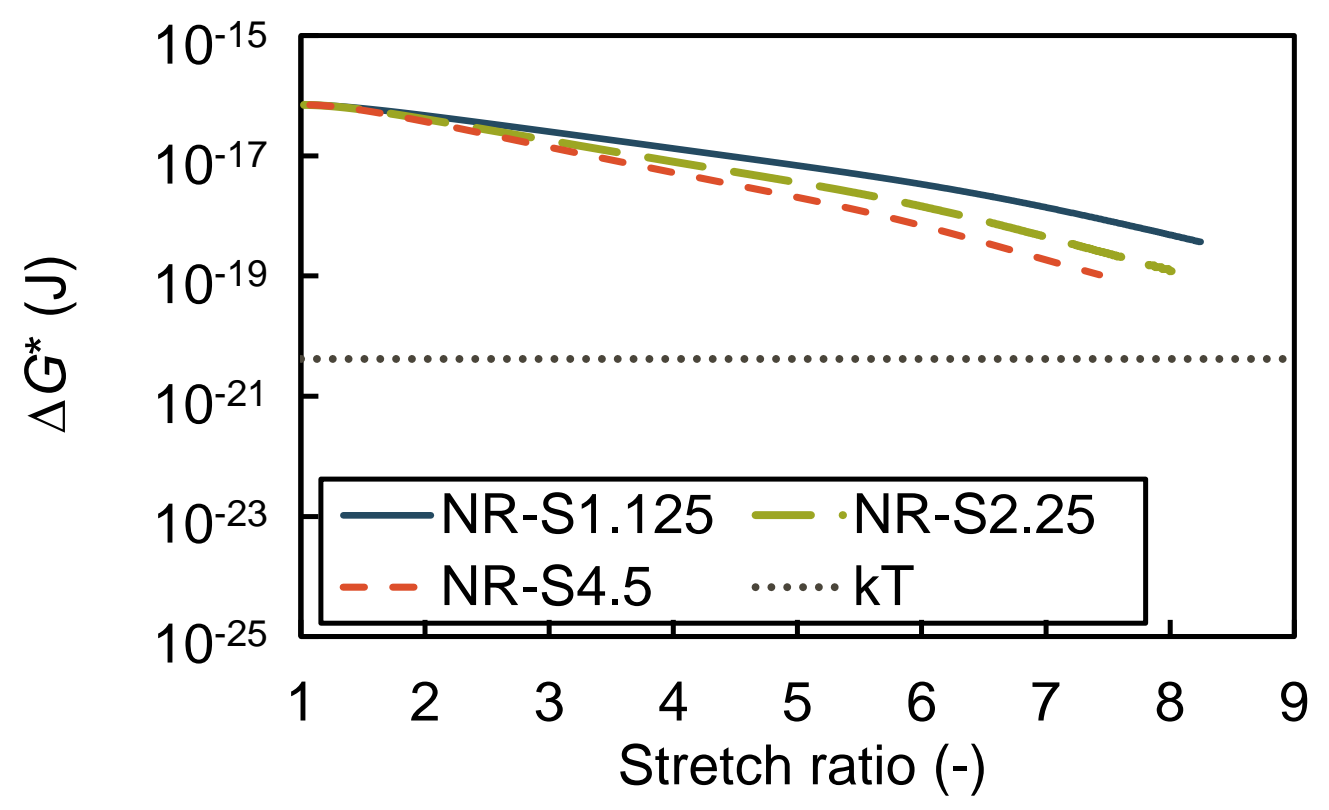




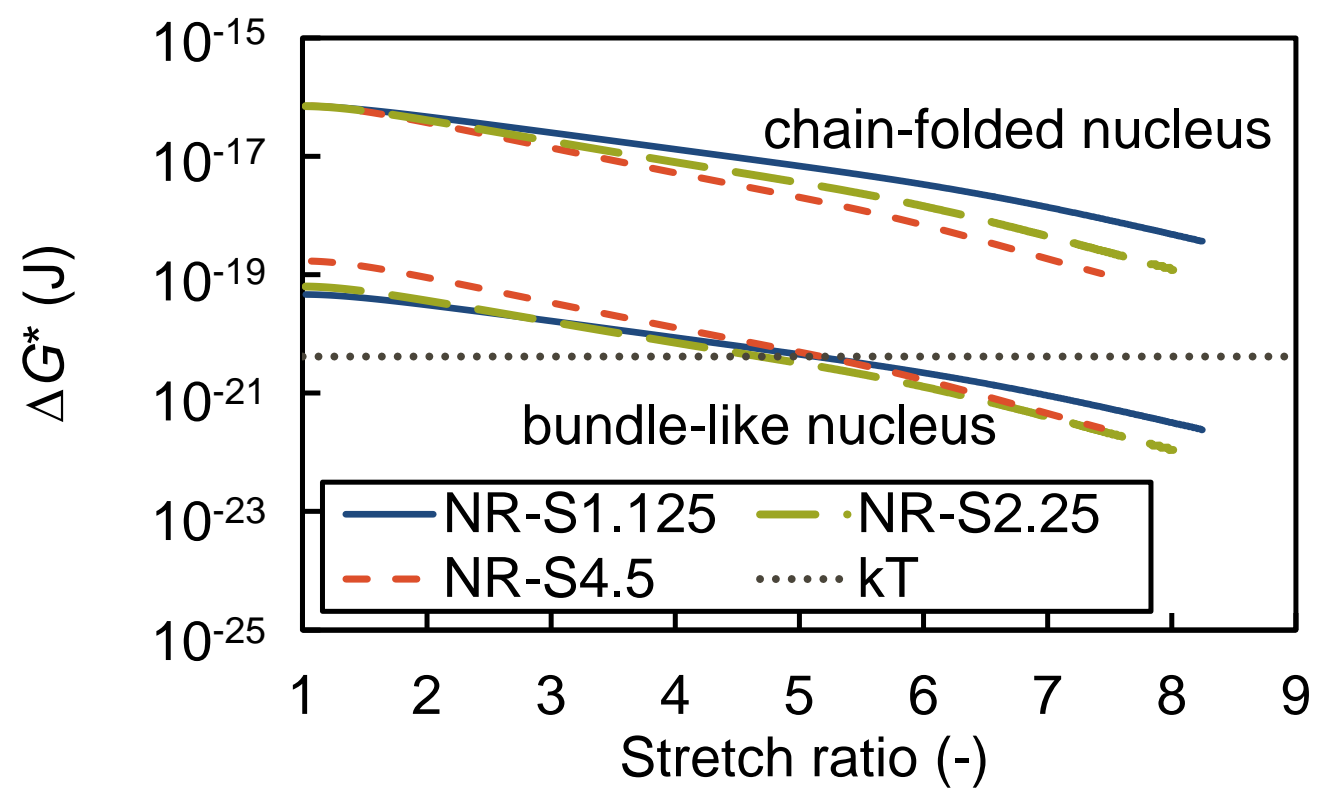




\section{Pen 京都大学}

Graphical abstract

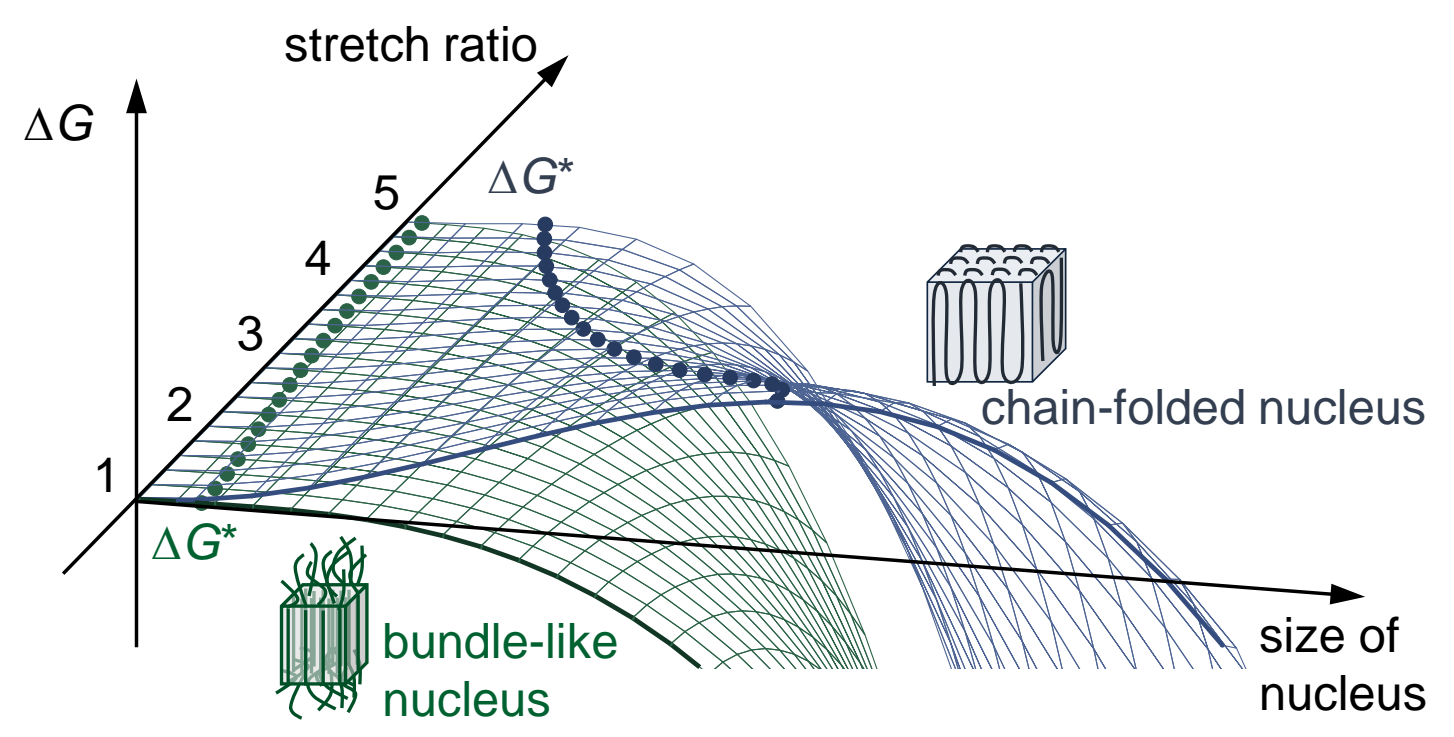

